\title{
Virtual Reality Navigation System for Prostate Biopsy
}

\author{
Lorenzo Rapetti \\ University of Illinois at Chicago, \\ United States \\ Politecnico di Milano, \\ Italy \\ lorenzo.rapetti@mail.polimi.it \\ Giancarlo Ferrigno \\ Politecnico di Milano, \\ Italy \\ giancarlo.ferrigno@polimi.it
}

\author{
Simone Crivellaro \\ University of Illinois at Chicago, \\ United States \\ crivesim@uic.edu
}

\author{
Craig Niederberger \\ University of Illinois at Chicago, \\ United States \\ craign@uic.edu
}

\author{
Elena De Momi \\ Politecnico di Milano, \\ Italy \\ elena.demomi@polimi.it \\ Cristian Luciano \\ University of Illinois at Chicago, \\ United States \\ clucia1@uic.edu
}

\begin{abstract}
Prostate cancer is the most common non-cutaneous cancer in America. Tumor detection involves non-invasive screening tests, but positive results must be confirmed by a prostate biopsy. About twelve random samples are obtained during the biopsy, which is a systematic procedure traditionally performed with trans-rectal ultrasound (TRUS) guidance to determine prostate location. Recently, methods of fusion between TRUS and preoperative MRI have been introduced in order to perform targeted biopsies aimed to reduce the number of samples to few suspicious areas. Since the TRUS displaces the prostate during the procedure, the preoperative MRI does not match patient anatomy. Therefore, complex MRI deformation algorithms are needed. However, despite the substantial increase in complexity and cost, there is no strong evidence that the TRUS-MRI fusion actually improves accuracy and surgical outcomes.

This paper presents an innovative virtual reality surgical navigation system for performing targeted prostate biopsies, without the need of the uncomfortable TRUS. Both biopsy needle and patient anatomy are constantly tracked by an electromagnetic tracking system that provides their 3D position and orientation with respect to the surgical bed. Multiple fiducial markers are placed on the patient skin (at the iliac crest and pubic bone) during MRI scanning. Once in the operative room, the surgeon is presented a stereoscopic 3D volumetric rendering and multiple orthogonal views of the patient anatomy, as well as a virtual representation of the tracked needle. After a simple registration
\end{abstract}

Permission to make digital or hard copies of all or part of this work for personal or classroom use is granted without fee provided that copies are not made or distributed for profit or commercial advantage and that copies bear this notice and the full citation on the first page. Copyrights for components of this work owned by others than the author(s) must be honored. Abstracting with credit is permitted. To copy otherwise, or republish, to post on servers or to redistribute to lists, requires prior specific permission and/or a fee. Request permissions from Permissions@acm.org.

VRST '17, November 8-10, 2017, Gothenburg, Sweden

(C) 2017 Copyright is held by the owner/author(s). Publication rights licensed to ACM.

ACM ISBN 978-1-4503-5548-3/17/11...\$15.00

https://doi.org/10.1145/3139131.3139162 process between the MRI and the tracker coordinate system, the navigation system guides the needle insertion in the patient perineum through several anatomical layers towards the biopsy targets.

\section{CCS CONCEPTS}

- Applied Computing $\rightarrow$ Life and medical sciences;

- Computer systems organization $\rightarrow$ Embedded systems;

\section{KEYWORDS}

Medical and health support, Prototyping/Implementation, Graphics/3D

\section{ACM Reference format:}

L. Rapetti, S. Crivellaro, E. De Momi, G. Ferrigno, C. Niederberger, C. Luciano. 2017. Virtual Reality Navigation System for Prostate Biopsy. In Proceedings of 23rd ACM Symposium on Virtual Reality Software and Technology, Gothenburg, Sweden, November 2017 (VRST 2017), 4 pages.

https://doi.org/10.1145/3139131.3139162

\section{INTRODUCTION}

Prostate cancer is the most common non-cutaneous cancer in American men, the most recent statistics available for the United States report 176,450 diagnoses and 27,681 cases of death in the 2013. Screening and early detection are key factors for the therapy of the cancer in order to have better patient outcomes. The prostate cancer screening involves the prostate-specific antigen blood test, the digital rectal exam, and multi-parametric MRI (mpMRI), but none of the existing tests is $100 \%$ accurate and an abnormal result need a prostate biopsy to determine the presence of cancer [Hoffman et al. 2002; Schro et al. 1998].

Prostate biopsy is the most accurate way to evaluate the suspicious cells inside the prostate gland; it consists in taking multiple samples from patient prostate to investigate under a microscope whether or not cancerous cells are present. Despite the importance and criticality of cancer detection, after the introduction of systematic biopsy procedure, there have been no innovative techniques granting an important progress in term of 
prostate cancer detection. However, over the past decade, navigation techniques in surgery have evolved in many fields; thanks to improved computer technologies and imaging methods, safer and less invasive procedures have been introduced [Mezger et al. 2013]. The introduction of virtual image-guided techniques resulted in good clinical results over the standard procedures reducing surgical time and mental load for the surgeon. In virtual image-based navigation systems, pre-operative images are registered to the patient position in the operative room, while medical instruments are tracked. Fusing the two information, the instruments can be visualized real-time in the same "virtual coordinate system" of the medical images facilitating the realization of the surgical planning.

Given the weakness of current biopsy navigation techniques, this project implements a virtual reality navigation system for prostate biopsy that has the potential to improve the accuracy and safety of the procedure replacing the TRUS real-time guidance with an electromagnetic tracking system (EMTS).

\section{BACKGROUND}

Prostate biopsy is a procedure whose aim is to investigate the presence of cancerous cells inside the prostate after an abnormal result in a screening test. To achieve this, a hollow needle is used to take about a dozen samples of tissue of the patient prostate under image guidance. The actual number of samples and the criterion with which the samples are taken can vary; the objective is to have a balance between high accuracy and specificity in one hand, and in the other the minimization of discomfort, pain and occurrence of complications for the patient (bleeding and infections).

\subsection{Systematic biopsy}

The most common procedure (12-core transrectal biopsy) consists in using a hollow needle that is inserted through the patient rectum in order to take 12 samples from different areas of the prostate under the guidance of real-time images provided by a transrectal ultrasound (TRUS) [Eicher et al. 2006]. The ultrasound images give information about the needle localization and the prostate anatomy, but they do not give any information about location of suspicious areas since most of the cancerous tissues are isoechoic. The success of this technique is due to the assumption that with a sufficient sampling size, it is possible to determine whether cancerous cells are present or not.

Despite considered as the "gold standard", systematic biopsy is a blind procedure that neglects the information that modern MRI techniques can provide about presence and location of cancerous cells [Kang et al. 2010; Shimofusa et al. 2005]. The absence of real-time images of tumor leads to sampling errors, unnecessary high number of samples, and false negative results [Serefoglu et al. 2013].

\subsection{Targeted Biopsy}

The advent of new image technologies has increased the number of visible cancer paving the way for developing protocols for targeted biopsy with several benefits over systematic prostate biopsy. The idea is that targeted biopsy can reduce the number of false negative avoiding an oversampling during biopsy procedure. Recently, methods of fusion between TRUS and preoperative MRI have been introduced in order to perform targeted biopsies whose guidance includes the discrimination information provided by MRI. Using as target the lesion detected by MRI, the TRUS tracks in real-time the position of the needle and it is fused to MRI images to have an intra-operative visualization of the target [Moore et al. 2013; Heijmink et al. 2006].

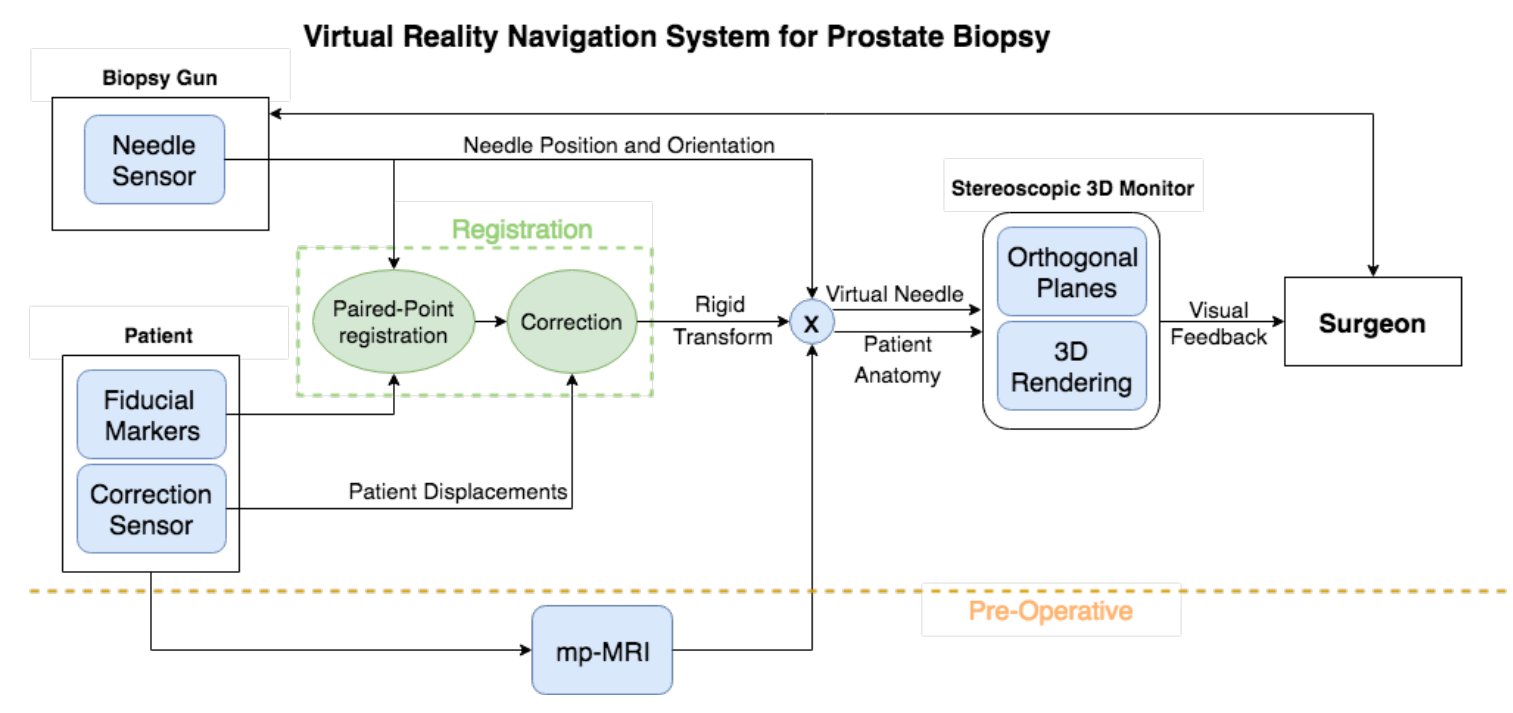

Figure 1. In the proposed navigation system, a rigid transform registers the position of the needle to the patient MRI. The surgeon is offered a real-time visual feedback of the position of the needle in the MRI volume 
What make this approach computationally costly are the elastic properties of prostate and surrounding tissues. The configuration of the prostate during the procedure and during the MRI scanning is different; the image fusion software has to be accurate and efficient in order to compute in real-time the deformation that maps the MRI volume in the ultrasound image, taking into account that the TRUS probe itself contribute to the tissues deformation.

In many cases, compared to the standard systematic biopsy, the fusion biopsy has shown in some cases improved detection rate and shorter surgical time [Pinto et al. 2011], but there is no strong evidence of those improvements. Moreover, this approach has significant higher costs, complexity and total time (which includes the image segmentation and the setting of the system) affecting both the surgeon mental load and surgical costs.

While the inclusion of MRI to perform a targeted biopsy represents an improvement over the systematic methods, the image fusion is a complex and inefficient process. For this reason, the aim of the project is to develop a navigation system that uses MRI to target suspicious areas, as a more efficient and accurate alternative to the TRUS-MRI fusion

\section{METHODS}

The proposed solution is a virtual reality navigation system for targeted biopsy in which a transperineal targeted biopsy is performed under the guidance of virtual images that replaces the TRUS guidance. The software was developed using LACE library, a $\mathrm{C}++$ software development kit for virtual/mixed reality applications, and the tracking system that was used is the Ascension 3D Guidance ${ }^{\circledR}$ tracking unit by NDI.

An overview of the system workflow is shown in Figure 1. The first step of the system is the acquisition of the model that will be the anatomical reference during the navigation. The model is obtained from pre-operative volumetric imaging (mp-MRI) of the patient pelvis that provides both anatomical information on the patient, and the localization of the suspicious areas that are possible targets for the biopsy. During the image acquisition, some fiducial markers are placed on the pelvis skin in correspondence of the iliac crest and pubic bone. Those markers are used to register the anatomical model to the instrument tracking system, and must remain on the patient until the biopsy is performed.

Before moving to the intra-operative phase, the surgeon can perform pre-surgical planning by choosing the location of the biopsy targets in the virtual coordinate system using the 3D interface offered by the application. During the biopsy, the position and orientation of the needle is real-time tracked by a sensor attached to the biopsy gun (needle sensor), while another sensor attached to the patient anatomy corrects the registration accordingly to pelvis movements during surgery (correction sensor). All the information is combined in a graphical interface that provides the surgeon a $3 \mathrm{D}$ visual feedback combining preoperative MRI of the patient with a virtual needle and preoperative plans.

\subsection{Registration}

Once in the surgical room, it is necessary to map in real-time the coordinates of the needle measured by the EMTS, to the patient anatomy model accordingly to the patient position. This registration process is executed right before the biopsy procedure, with the patient in the surgical lithotomy position.

To obtain the mapping transform the tracker is turned on, and the markers are touched with a sensor in order to determine their position in the EMTS coordinate system. At this moment, the positions of the markers are known both in virtual and in EMTS coordinate system, so a paired-point registration algorithm is used to determine the rigid transform matrix that matches the two systems. The registration algorithm that has been chosen is widely used for computer aided surgery [Sorkine-Hornung et al. 2017]. It is based on least square error minimization, and it uses the singular value decomposition (SVD) of the covariance matrix of the two point distribution (in virtual and EMTS coordinates) to compute the transform matrix that registers one coordinate system to the other. The SVD is computed using the two-sided Jacobi iteration method [Luk et al. 1989] that is implemented in the $\mathrm{C}++$ template library Eigen.

In order to avoid performing multiple registrations if the patient moves, the correction sensor attached to the patient pelvis measures patient displacements and corrects the registration transform preserving the matching between the two systems of coordinates.

\subsection{Navigation}

During the procedure, a real-time visual feedback is provided to the surgeon through a stereoscopic 3D monitor. The graphic interface displays virtual images of needle and internal anatomy both using 3D rendering and multiple orthogonal view of the patient anatomy (as shown in Figure 2).

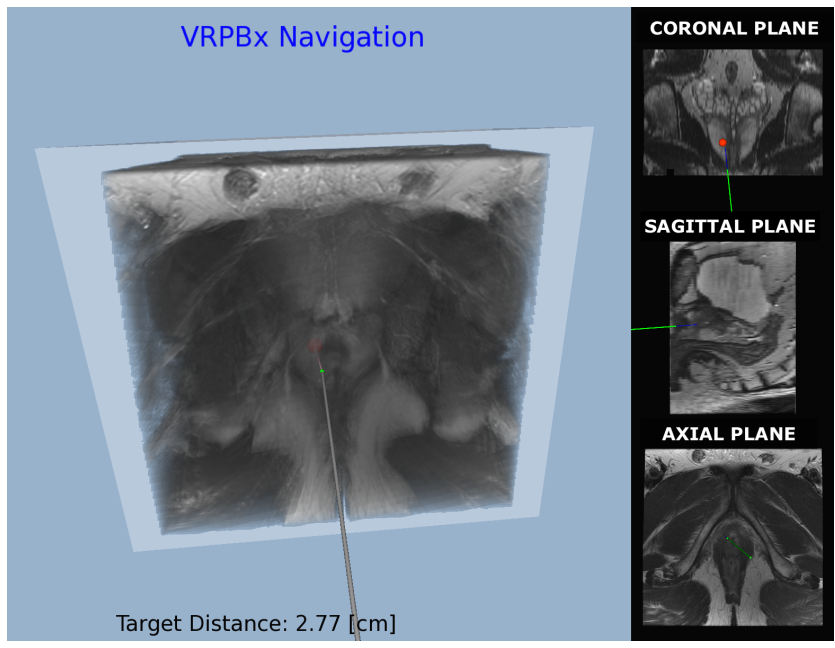

Figure 2. During the navigation, the screen offers a stereoscopic $3 \mathrm{D}$ volumetric rendering and multiple orthogonal views of the patient anatomy. In both $2 \mathrm{D}$ and $3 D$ views, the position of the needle and the target point are visible. 
The rendering of the volumetric image can be done with three different modalities: volume sliced, volume ray casting, and visualizing the three orthogonal planes centered on the needle tip. The transfer function and transparency can be interactively adjusted by the user. The system also provides the possibility to clip the volume with a plane in order to have a better view of internal anatomy.

Furthermore, the navigation system gives the surgeon additional information to guide the surgeon accordingly to intraoperative performances and pre-operative planning. Those features include the position of the cores that were previously taken, the distance between the needle and the target, the shot range of the needle, and the direction from the needle tip to the target point. The graphical interface is intuitive and flexible to adapt to patient condition and surgeon preferences. The information provided allows the surgeon to efficiently perform a targeted biopsy without an overload of information.

The EMTS tracks the 3D position and orientation of the needle in the surgical field during the procedure replacing the TRUS for real-time tracking. The use of EMTS prevents the tissue deformation caused by the TRUS probe.

Moreover, with the TRUS the position of the needle is derived from the surrounding visible tissues only when the needle is in the visual field of the ultrasound. With the EMTS the position of the needle is always known once the registration is done. As consequence, the virtual reality navigation system for prostate biopsy offers a guidance not only when the needle is inside the patient anatomy, but also while the needle is outside the patient optimizing perineum access.

\section{RESULTS AND CONCLUSIONS}

The virtual reality navigation system for prostate biopsy has been preliminary tested by a cohort of experienced urology surgeons using a ballistic gel phantom to perform the registration and navigation process, observing the proposed graphic interface exploring the different modalities for guided and targeted navigation. The urologists have underlined the weakness of systematic approach and MRI-TRUS image fusion techniques, for this reason they agree about the need of applying modern technologies to move towards intuitive and easy-to-use methods for targeted biopsy expressing enthusiasm in the possibility to replace the TRUS with the proposed tracking technique.

They have observed that the proposed graphical interface offers a complete anatomical information for prostate biopsy navigation thanks to the combination of a $3 \mathrm{D}$ environment with the $2 \mathrm{D}$ orthogonal views. The enhanced virtual visual feedback gives the surgeon the opportunity of performing a targeted biopsy with short pre-processing time, guided by a simple interface. Furthermore, they have positively highlighted the unique opportunity offered by the system to aligning the needle with the target before inserting it through the perineum significantly reducing the surgical time.

The proposed solution is an innovative and unique approach for prostate biopsy combining an EMTS and virtual reality to replace the traditional TRUS real-time instrument guidance.

\section{REFERENCES}

K. Eichler, S. Hempel, J. Wilby, L. Myers, L. M. Bachmann, and J. Kleijnen, "Diagnostic Value of Systematic Biopsy Methods in the Investigation of Prostate Cancer: A Systematic Review," J. Urol., vol. 175, no. 5, pp. 1605-1612, 2006.

S. W. T. P. J. Heijmink, H. van Moerkerk, L. A. L. M. Kiemeney, J. A. Witjes, F. Frauscher, and J. O. Barentsz, "A comparison of the diagnostic performance of systematic versus ultrasound-guided biopsies of prostate cancer," Eur. Radiol., vol. 16, no. 4, pp. 927-938, 2006.

R. M. Hoffman, F. D. Gilliland, M. Adams-Cameron, W. C. Hunt, and C. R. Key, "Prostate-specific antigen testing accuracy in community practice.," BMC Fam. Pract., vol. 3, p. 19, 2002.

P. Kang, M. Liao, M. R. Wester, J. S. Leeder, and R. E. Pearce, "Multiparametric MRI of Prostate Cancer: An Update on State-of- theArt Techniques and Their Performance in Detecting and Localizing Prostate Cancer," Ratio, vol. 36, no. 3, pp. 490-499, 2010.

F. T. Luk and H. Park, "A Proof of Convergence for two Parallel Jacobi SVD Algorithms,” IEEE Trans. Comput., vol. 38, no. 6, pp. 806-811, 1989.

U. Mezger, C. Jendrewski, and M. Bartels, "Navigation in surgery," Langenbeck's Arch. Surg., vol. 398, no. 4, pp. 501-514, 2013.

C. M. Moore et al., "Image-guided prostate biopsy using magnetic resonance imaging- derived targets: A systematic review," Eur. Urol., vol. 63, no. 1, pp. 125-140, 2013.

P. A. Pinto et al., "Magnetic Resonance Imaging / Ultrasound Fusion Guided Prostate Biopsy Improves Cancer Detection Following Transrectal Ultrasound Biopsy and Correlates with Multiparametric Magnetic Resonance Imaging," J. Urol., vol. 186, no. 4, pp. 1281-1285, 2011.

F. H. Schro, P. Van Der, P. Beemsterboer, B. Kruger, and J. Rietbergen, "Evaluation of the Digital Rectal Examination as a Screening Test for Prostate Cancer," vol. 90, no. 23, pp. 1817-1823, 1998.

E. C. Serefoglu, S. Altinova, N. S. Ugras, E. Akincioglu, E. Asil, and M. D. Balbay, "How reliable is 12-core prostate biopsy procedure in the detection of prostate cancer?" Can. Urol. Assoc. J., vol. 7, no. 5-6, pp. E293-8, 2013.

R. Shimofusa et al., "Diffusion-weighted imaging of prostate cancer," J. Comput. Assist. Tomogr., vol. 29, no. 2, pp. 149-153, 2005.

O. Sorkine-hornung and M. Rabinovich, "Least-Squares Rigid Motion Using SVD," no. 3, pp. 1-5, 2017 